\title{
The psychiatric profile of the U.S. patient population across age groups
}

\author{
Mohammad Sami Walid $^{1 *}$, Nadezhda Zaytseva ${ }^{2}$, William Perez ${ }^{3}$ \\ ${ }^{1}$ Postgraduate Year One (PGY1) Resident, Internal Medicine, Kingsbrook Jewish Medical Center, Brooklyn, USA \\ ${ }^{2}$ Faculty Member, Kuban State Medical University, Krasnodar, Russia \\ ${ }^{3}$ Personal Life Consultant, PARLEY Personal Life Consulting Services, Macon, USA \\ Email: ${ }^{*}$ mswalid@yahoo.com
}

Received 10 January 2012; revised 2 March 2012; accepted 15 March 2012

\begin{abstract}
Introduction: As the U.S. population undergoes continuous shifts the population's health profile changes dynamically resulting in more or less expression of certain psychiatric disorders and utilization of healthcare resources. In this paper, we analyze national data on the psychiatric morbidity of American patients and their summated cost in different age groups. Methods: The latest data (2009) on the number of hospital discharges and national bill (hospital charges) linked with psychiatric disorders were extracted from the Nationwide Inpatient Sample (NIS). Results: National data shows that mood disorders are the largest diagnostic category in terms of percentage of psychiatric-related discharges in the 1 - 17 years age group. The proportion decreases gradually as age progresses while delirium, dementia, amnestic and other cognitive disorders increase exponentially after 65 years of age. Schizophrenia and other psychotic disorders as well as alcohol and substance-related disorders peak in the working age groups (18 - 64 years). From an economic point of view, mood disorders in the 18 - 44 age group has the highest national bill ( $\$ 5.477$ billion) followed by schizophrenic and other psychotic disorders in the same age group (\$4.337 billion) and mood disorders in the 45 - 64 age group ( $\$ 4.310$ billion). On the third place come schizophrenic and other psychotic disorders in the 45 - 64 age group (\$3.931 billion). Conclusion: This paper illustrates the high cost of psychiatric care in the U.S., especially the large fraction of healthcare money spent on working-age patients suffering from mood disorders. This underlines psychiatric cost-efficiency as a vital topic in the current healthcare debate.
\end{abstract}

Keywords: Psychiatric; Diagnosis; Hospital Discharges; Hospital Charges

${ }^{*}$ Corresponding author.

\section{INTRODUCTION}

The population of the United States (U.S.) is a vibrant population with constant influx of new immigrants and continuous internal restructuring due to fluctuations in birth and death rates and dynamic ethnic shifts. Latest data shows that life expectancy in the U.S. was 77.9 in 2007 [1], reached 78.2 in 2009 [2] and is projected to be around 80.1 in 2020 [3]. Furthermore, 39.4 million people 60 years and older (July 2008) live in the U.S. [1]. By 2030, the number of U.S. adults aged 65 or older is predicted to reach about 71 million [4].

As a collateral manifestation of these demographic changes, the population's health profile changes and the resultant utilization of healthcare resources responds to more or less expression of certain diagnostic categories, including psychiatric disorders. In this paper, we analyze national data on the psychiatric morbidity of American patients and their summated cost in different age groups in order to draw attention to the importance of costanalysis in psychiatric research. Determining the most cost-effective interventions for psychiatric disorders represents one key consideration into the broader task of public health decision-making and priority-setting with regard to investing resources in mental health [5-7].

\section{METHODS}

The latest data (2009) on the number of discharges and amount of expenses linked with psychiatric disorders were extracted from an online query system that grants access to the largest set of publicly available all-payer health care databases - Nationwide Inpatient Sample (NIS) of the Healthcare Cost and Utilization Project (HCUP), the Agency for Healthcare Research and Quality (AHRQ).

We studied the diagnostic and economic psychiatric profile of American patients in relationship to age, which we defined as the spectrum of discharges or national expenses associated with different psychiatric categories across age groups. Psychiatric diagnoses were clustered 
into a manageable number of clinically meaningful categories according to the Clinical Classifications Software (CCS) developed at the AHRQ.

The HCUPnet presents hospital charges associated with the principal diagnosis (the condition that is the chief reason for the hospital stay, as determined after evaluation during this stay) and uses the term "national bill" for summated hospital charges on a national level. Expenses going to physician consultation services are not provided by that website.

\section{RESULTS}

National data shows that mood disorders are the largest diagnostic category in terms of the total number of hospital discharges in the $1-17$ years age group (Figure 1). Since the 18 - 44 age group is the largest age group of the population it has the largest numbers of patients with mood disorders $(436,268)$, psychotic disorders $(201,274)$ and substance-related disorders $(137,204)$. Hospital discharges referable to alcoholrelated disorders are highest in absolute numbers (Figure 2) in the 45 - 64 age group $(135,164)$ then in the $18-44$ age group $(110,897)$.

Percentagewise, mood disorders constitute the largest psychiatric diagnosis related to hospital discharges in the
1 - 17 years age (Figure 3). The mood disorders proportion decreases gradually as age progresses while delirium, dementia, amnestic and other cognitive disorders increase exponentially after 65 years of age. Moreover, attentiondeficit, conduct, and disruptive behavior disorders and impulse control disorders are specific to the younger age group. On the other hand, schizophrenia and other psychotic disorders as well as alcohol and substance-related disorders peak in the working age groups (18 - 64 years).

From an economic point of view, mood and psychotic disorders in working-age patients take the biggest toll on the healthcare budget (Figure 4). Mood disorders in the 18 - 44 age group has the highest national bill (\$5.477 billion) followed by schizophrenic and other psychotic disorders in the same age group ( $\$ 4.337$ billion) and mood disorders in the 45 - 64 age group ( $\$ 4.310$ billion). On the third place come schizophrenic and other psychotic disorders in the 45 - 64 age group (\$3.931 billion). Otherwise, alcohol-related disorders in the 45 - 64 age group cost the healthcare budget $\$ 2.327$ billion and screening for mental disorders and substance abuse in the same age group costs $\$ 2.228$ billion; though the cost of substance-related disorders is highest in the 18 - 44 age group and amounts to $\$ 1.624$ billion. Unsurprisingly, delirium, dementia, amnestic and other cognitive disorders

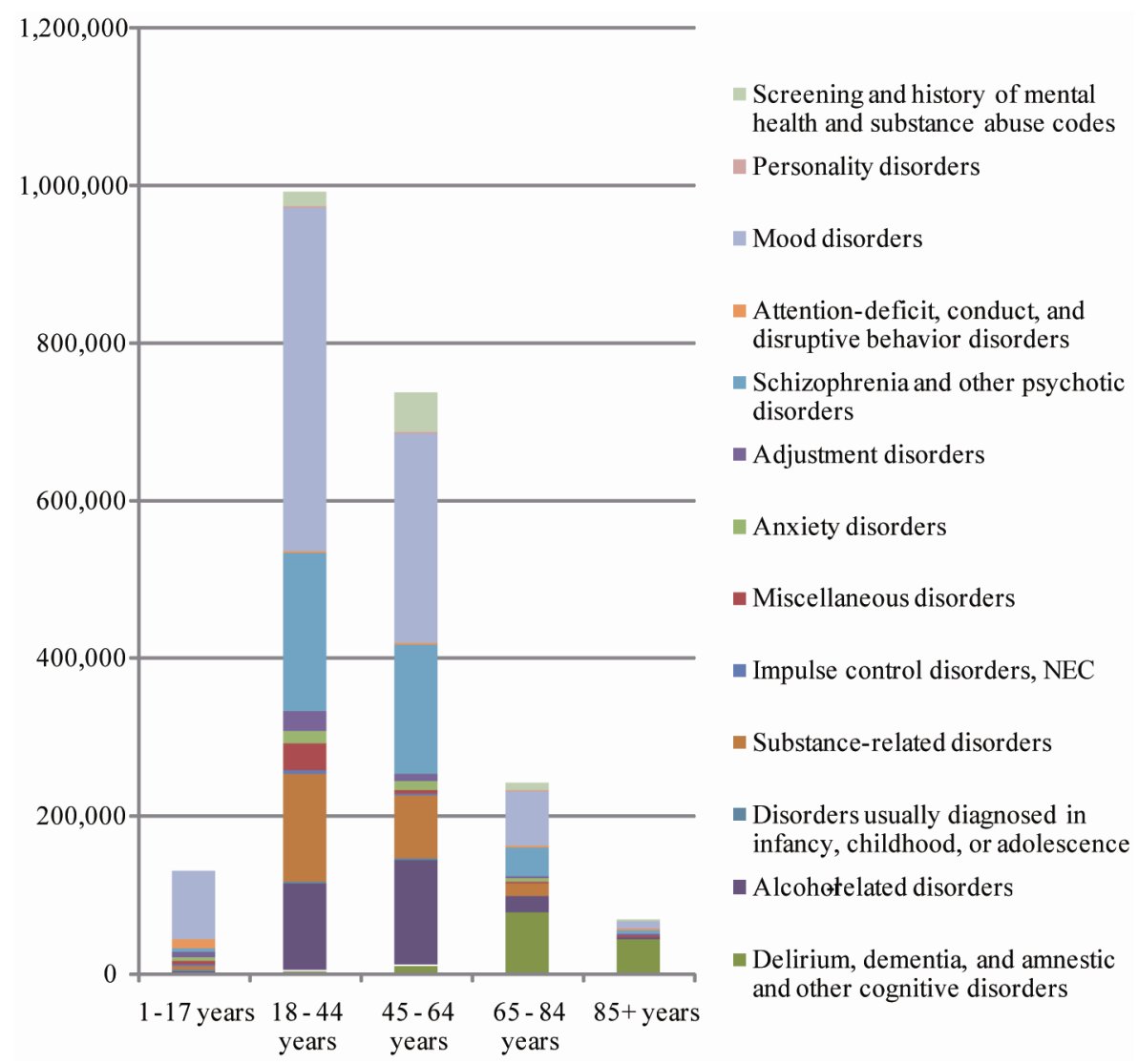

Figure 1. Number of discharges related to psychiatric categories in different age groups. 


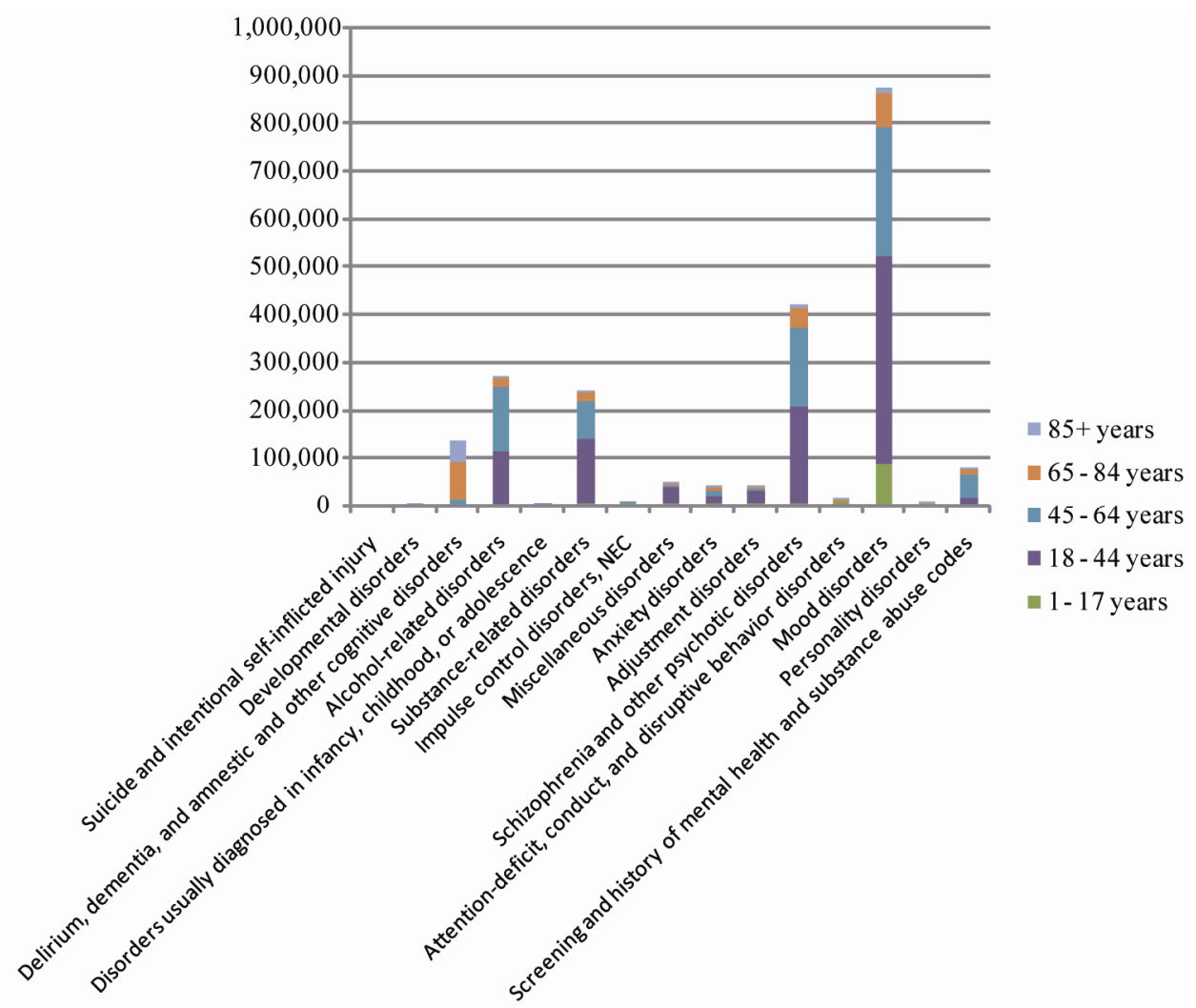

Figure 2. Number of discharges of different age group patients per psychiatric category.

have the highest bill in the 65 - 84 age group totaling $\$ 1.725$ billion.

Studying the national bill trends of mood disorders and cognitive disorders in certain age groups we find that from 1997 to 2009 the national bill of mood disorders increased 2.4 fold (from 2,236,550,148 to 5,477,797,677) in the 18 - 44 age group and 3.6 fold $(1,197,429,873$ to $4,310,815,222)$ in the 45 - 64 age group. On the other hand, in the senior age groups the increase tempo in national bill of cognitive disorders from 1997 to 2009 was nearly similar in the 65 - 84 and $85+$ age groups. Thus, the national bill of cognitive disorders in the early-old age group increased 2.2 fold from \$753,565,509 in 1997 to $\$ 1,725,414,702$ in 2009 and in the 85+ age group the increase was 2.7 fold from $\$ 315,357,553$ in 1997 to $\$ 873,628,602$ in 2009 (Figure 5).

\section{DISCUSSION}

Psychiatric illnesses are common and can be costly and debilitating. Mood disorders (depression and bipolar disease) in particular are frequently encountered. In the U.S, approximately $9.1 \%$ of the population meets the diagnostic criteria for current depression while $4.1 \%$ meet the criteria for major depression [8]. Reflecting that reality, $10 \%$ of the population is reported to be on antidepressants-considered the most commonly prescribed medications in the U.S. [9]. Analysis of national data shows that a large fraction of healthcare money (\$9.778 billion) is spent on working-age patients suffering from mood disorders (705 thousand). This makes screening protocols for psychiatric morbidities an economic necessity and an investment in the healthcare of the working population.

From their part, screening and history of mental health and substance abuse, despite representing a small percentage of hospital discharges, also consume a signifycant proportion of hospital resources to the benefit of working age and early-old age groups. Screening is a preliminary process of determining whether an individual does or does not warrant further attention at the current time in regard to a particular problem. Screening does not replace an official psychiatric assessment and therefore does not yield a diagnosis. It facilitates the early detection of people at immediate risk and assists in the identification of people with complicated history.

In this context, an Employer's Guide to Workplace Substance Abuse: Strategies and Treatment Recommendations was developed by the National Business Group on Health (NGBH) to help employers understand substance abuse, the prevalence of this problem among working adults and the costs related to it [10]. The Substance Abuse and Mental Health Services Administration 


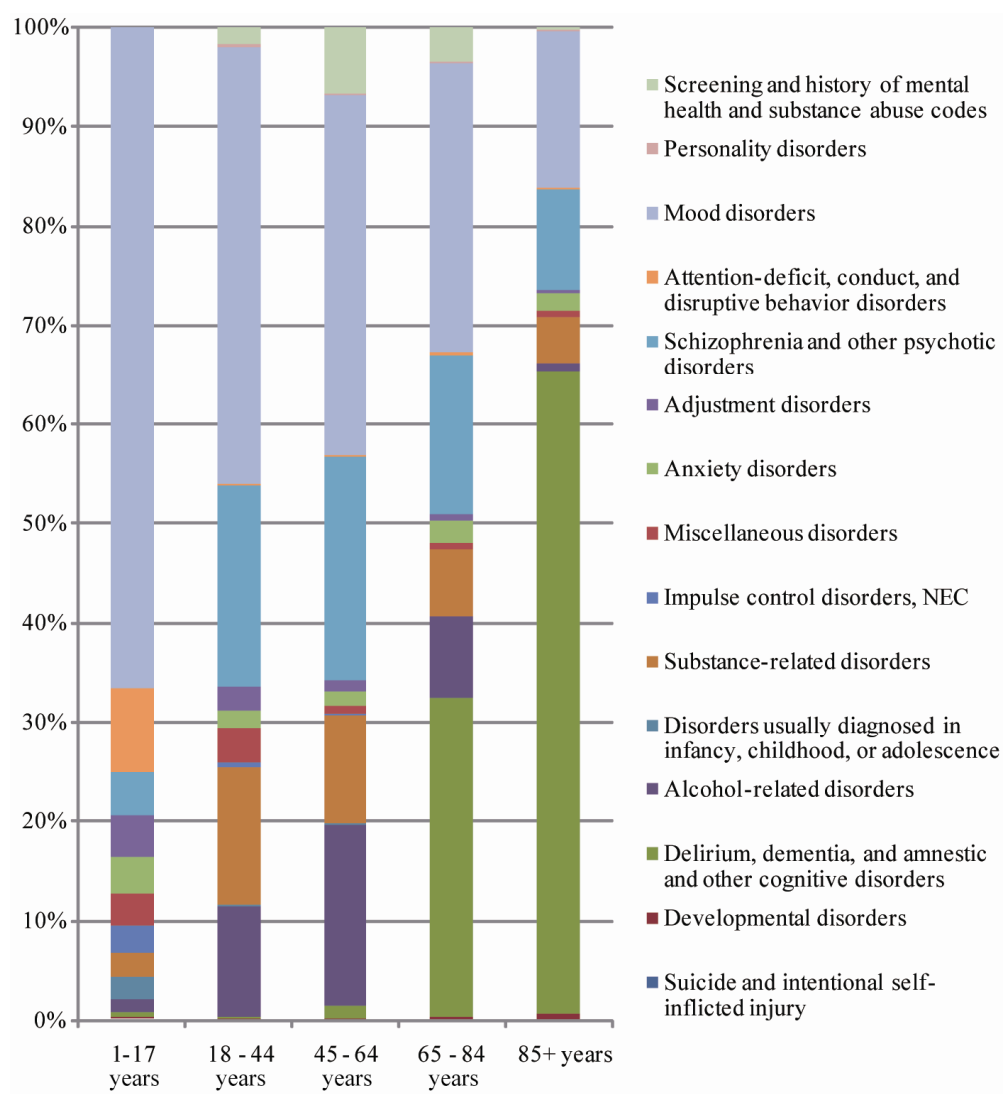

Figure 3. The diagnostic profile of American hospital discharges related to psychiatric morbidity.

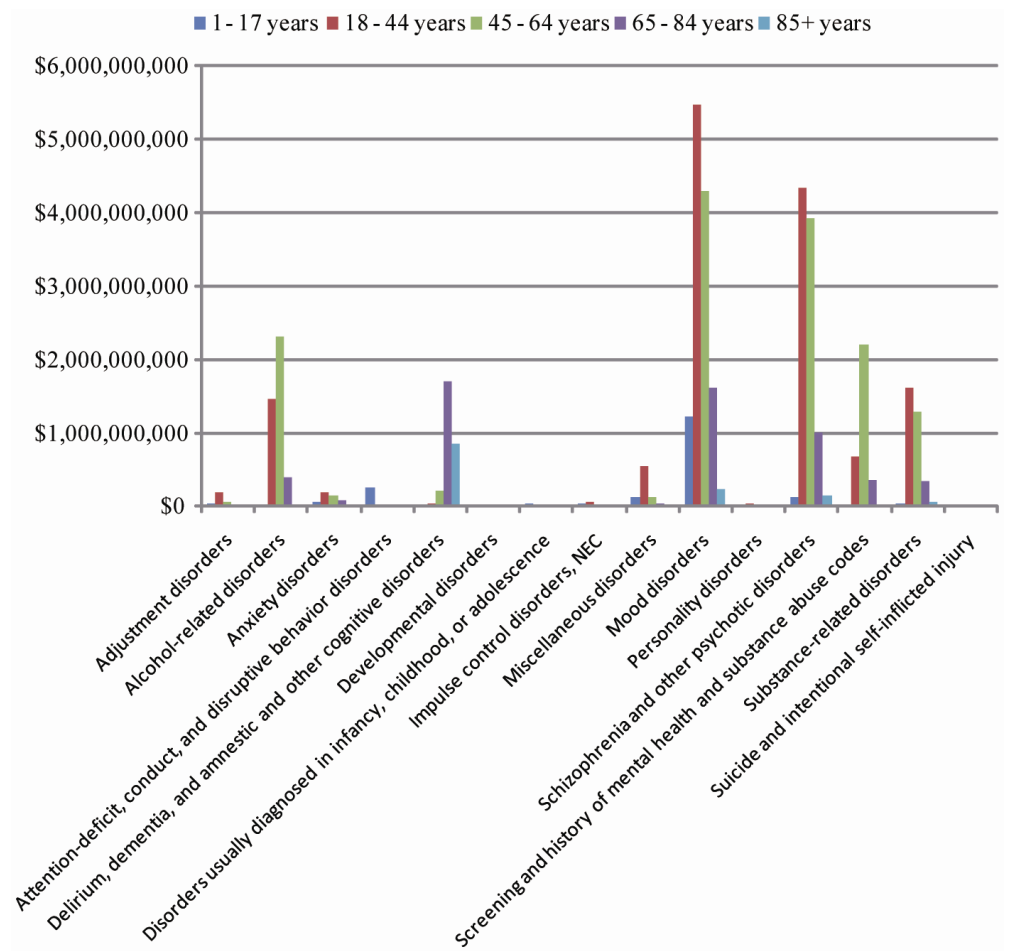

Figure 4. The economic profile of American hospital discharges related to psychiatric morbidity. 


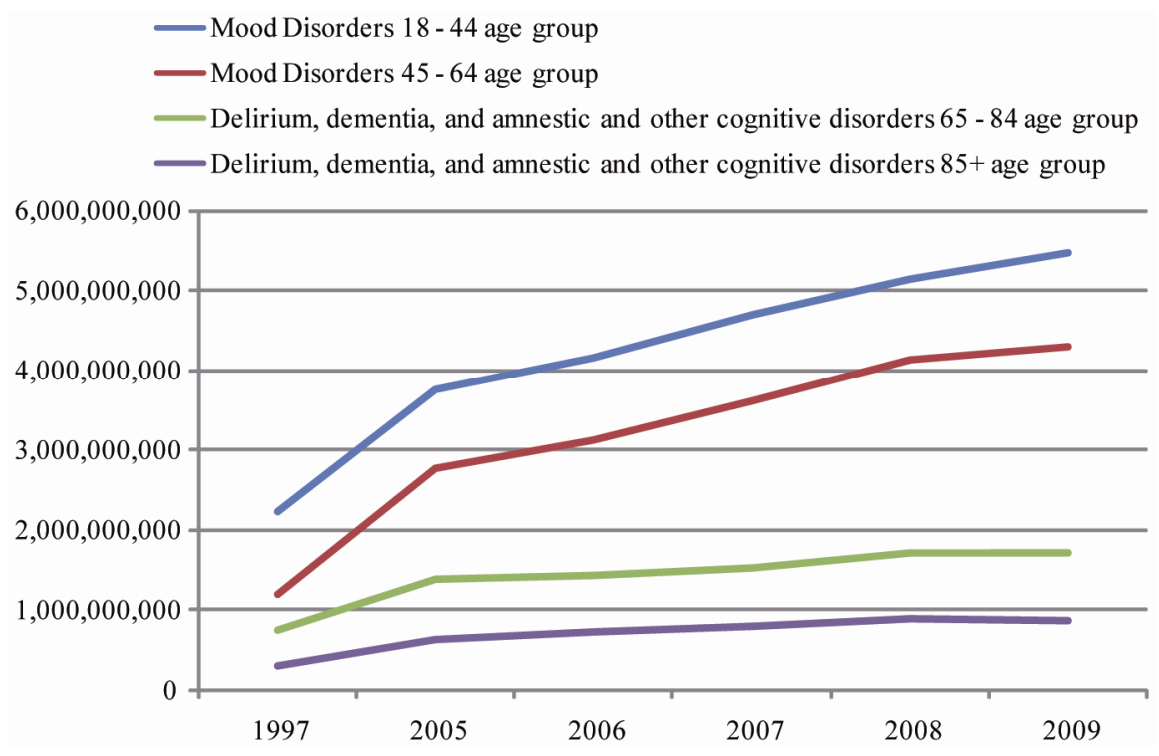

Figure 5. The increase in the national bill of mood and cognitive disorders in the American population.

(SAMHSA) data shows that 20 million adults were classified as having problems with substance dependence or abuse in 2007, approximately 12 million (60\%) of them were employed full time [11]. Our study shows that substance-related disorders are a big problem in working age groups (18 - 64 years) with an incredible cost verging on $\$ 2.934$ billion.

Additionally, it is estimated that more than half of the adult U.S. population drinks alcohol in a moderate or heavy way [12]; yet alcohol-related psychiatric disorders are not the leading psychiatric diagnosis connected with hospital discharges in the U.S. Alcohol abuse has immediate effects that can increase the risk of many harmful health conditions. The summated national hospital expenses attributed to alcohol-related disorders in all age groups in our study total $\$ 4.241$ billion. In fact, alcohol abuse has far-reaching consequences on the productivity and social life of the individual. A study by the CDC [13] estimated that excessive alcohol consumption actually costs the United States $\$ 223.5$ billion (2006). The costs largely resulted from losses in workplace productivity (72\%), health care expenses (11\%), legal and criminal justice expenses (9\%), and motor vehicle accidents (6\%).

The most dreaded cognitive disorder, Alzheimer's disease, is at present the sixth leading cause of death in the U.S. [2]. The rate for Alzheimer's disease increased dramatically due to improvements in diagnosis and awareness of the condition within the medical community. Currently, specialists believe that Alzheimer's disease has an insidious course beginning long before family and friends notice a dramatic change in the patient's memory and behavior [14]. By the time an official diagnosis is established, the person's function is usually significantly impaired and resistant to rehabilitation.
In summary, this paper illustrates the high cost of psychiatric care in the U.S., especially the large fraction of healthcare money spent on working-age patients suffering from mood disorders which makes psychiatric costefficiency a vital topic in the current healthcare debate.

\section{REFERENCES}

[1] Google Public Data Explorer (2012) Population in the U.S.

http://www.google.com/publicdata/explore?ds=kf7tgg1uo 9ude_\&met_y=population\&idim=country:US\&dl=en\&hl Een\&q=population+of+the+united+states

[2] Kochanek, K.D., Xu, J.Q., Murphy, S.L., et al. (2011) Deaths: Preliminary data for 2009.

http://www.cdc.gov/nchs/data/nvsr/nvsr59/nvsr59_04.pdf

[3] Department of Economic and Social Affairs Population Division (2007) World population prospects: The 2006 revision. http://www.un.org/esa/population/ordering.htm

[4] National Center for Chronic Disease Prevention and Health Promotion (2009) Healthy aging: Improving and extending quality of life among older Americans.

http://www.cdc.gov/nccdphp/publications/aag/pdf/health y_aging.pdf

[5] Hutubessy, R., Chisholm, D. and Edejer, T.T. (2003) Generalized cost-effectiveness analysis for national-level priority-setting in the health sector. Cost Effectiveness and Resource Allocation, 2003, 1, 8. doi:10.1186/1478-7547-1-8

[6] Chisholm, D., Sanderson, K., Ayuso-Mateos, J.L. and Saxena, S. (2004) Reducing the global burden of depression: Population-level analysis of intervention cost-effectiveness in 14 world regions. The British Journal of Psychiatry, 184, 393-403. doi:10.1192/bjp.184.5.393

[7] Chisholm, D., Van Ommeren, M., Ayuso-Mateos, J.L. and Saxena, S. (2005) Cost-effectiveness of clinical in- 
terventions for reducing the global burden of bipolar disorder. The British Journal of Psychiatry, 187, 559-567. doi:10.1192/bjp.187.6.559

[8] Centers for Disease Control and Prevention (2010) Current depression among adults-United States, 2006 and 2008. Morbidity and Mortality Weekly Report, 59, 12291235.

[9] Olfson, M. and Marcus, S.C. (2009) National patterns in antidepressant medication treatment. Archives of General Psychiatry, 66, 848-856.

doi:10.1001/archgenpsychiatry.2009.81

[10] Slavit, W.I., Reagin, A. and Finch, R.A. (2009) An employer's guide to workplace substance abuse: Strategies and treatment recommendations. Center for Prevention and Health Services, Washington DC.

[11] Department of Health and Human Services (2008) Re- sults from the 2007 national survey on drug use and health: National findings.

http://www.samhsa.gov/data/nsduh/2k7nsduh/2k7Results. $\underline{\mathrm{htm}}$

[12] Mann, D. (2010) More Americans drinking (alcohol). http://www.cnn.com/2010/HEALTH/07/20/americans.dri nking.alcohol.study/index.html

[13] Bouchery, E.E., Harwood, H.J., Sacks, J.J., Simon, C.J. and Brewer, R.D. (2011) Economic costs of excessive alcohol consumption in the U.S., 2006. American Journal of Preventive Medicine, 41, 516-524. doi:10.1016/j.amepre.2011.06.045

[14] Galimberti, D. and Scarpini, E. (2011) Progress in Alzheimer's disease. Journal of Neurology, 259, 201-211. doi:10.1007/s00415-011-6145-3 


\section{Appendix: Psychiatric Categories According to the Clinical Classification Software}

Adjustment disorders

3090309130922309233092430928309293093

30943098230983309893099

Anxiety disorders

2938430000300013000230009300103002030021 3002230023300293003300530089300930803081 308230833084308930981313031313132131322 31333138231383

Attention-deficit, conduct, and disruptive behavior disorders

3120031201312023120331210312113121231213

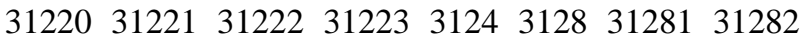
3128931293138131400314013141314231483149

Delirium, dementia, and amnestic and other cognitive disorders

29002901029011290122901329020290212903 2904029041290422904329082909293029312940 2941294102941129420294212948294931003102 3108310813108931093310331133111331193312 33182797

Developmental disorders

307030793150031501315023150931513152 31531315323153431535315393154315531583159 317318031813182319 V400 V401

Disorders usually diagnosed in infancy, childhood, or adolescence

2990029901299102991129980299812999029991

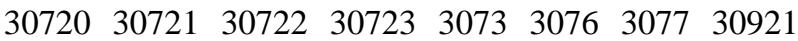
31323313893139

Impulse control disorders, NEC

31230312313123231233312343123531239

Miscellaneous disorders

293892939300113001230013300143001530016 300193006300730081300823021302230233024 302503025130252302533026302703027130272 3027330274302753027630279302813028230283 302843028530289302930603061306230633064 30650306513065230653306593066306730683069 307130740307413074230743307443074530746 3074730748307493075030751307523075330754 $\begin{array}{llllllll}30759 & 30780 & 30781 & 30789 & 3101 & 316 & 64840 & 64841\end{array}$ 648426484364844 V402 V403 V4031 V4039 V409 V673

Mood disorders

2938329600296012960229603296042960529606 2961029611296122961329614296152961629620 2962129622296232962429625296262963029631 2963229633296342963529636296402964129642 2964329644296452964629650296512965229653
2965429655296562966029661296622966329664 296652966629672968029681296822968929690 296993004311

Personality disorders

301030110301113011230113301203012130122 30133014301503015130159301630173018130182 3018330184301893019

Schizophrenia and other psychotic disorders

2938129382295002950129502295032950429505 2951029511295122951329514295152952029521 2952229523295242952529530295312953229533 2953429535295402954129542295432954429545 2955029551295522955329554295552956029561 2956229563295642956529570295712957229573 2957429575295802958129582295832958429585 29590295912959229593295942959529702971 2972297329782979298029812982298329842988 2989

Alcohol-related disorders

29102911291229132914291529182918129182 291892919303003030130302303033039030391 303923039330500305013050230503760719800

Substance-related disorders

29202921129212292229281292822928329284 292852928929293040030401304023040330410 3041130412304133042030421304223042330430 3043130432304333044030441304423044330450 3045130452304533046030461304623046330470 3047130472304733048030481304823048330490 3049130492304933052030521305223052330530 3053130532305333054030541305423054330550 3055130552305533056030561305623056330570 3057130572305733058030581305823058330590 3059130592305936483064831648326483364834 655506555165553760727607376075779596500 965019650296509 V6542

Suicide and intentional self-inflicted injury

E9500 E9501 E9502 E9503 E9504 E9505 E9506 E9507 E9508 E9509 E9510 E9511 E9518 E9520 E9521 E9528 E9529 E9530 E9531 E9538 E9539 E954 E9550 E9551 E9552 E9553 E9554 E9555 E9556 E9557 E9559 E956 E9570 E9571 E9572 E9579 E9580 E9581 E9582 E9583 E9584 E9585 E9586 E9587 E9588 E9589 E959 V6284

Screening and history of mental health and substance abuse codes

3051305103051130512305133339235754255 5353535305353157105711571257137903 V110 V111 V112 V113 V114 V118 V119 V154 V1541 V1542 V1549 V1582 V6285 V663 V701 V702 V7101 V7102 V7109 V790 V791 V792 V793 V798 V799 